\title{
Eine einfache Berechnung der Abbremsung schneller H-Atome durch kaltes H-Gas*
}

\author{
J. SCHÄFER und E. TREFFTZ \\ Max-Planck-Institut für Physik und Astrophysik, München \\ (Z. Naturforsch. 25 a, 863-867 [1970]; eingegangen am 13. April 1970)
}

\begin{abstract}
In order to make a simple estimate of the thermalization of neutralized solar wind particles, classical elastic energy transfer cross sections are calculated for the $\mathrm{H}-\mathrm{H}$ scattering process. A very simple approximation is also used for the macroscopic decelleration process. The estimated distance over which the hydrogen atoms lose half of their kinetic energy is $0.32 \cdot 10^{18} \mathrm{~cm}$ and $24 \cdot 10^{18} \mathrm{~cm}$, for a starting velocity of $50 \mathrm{~km} / \mathrm{sec}$ and $400 \mathrm{~km} / \mathrm{sec}$, resp., and for interstellar hydrogen density of $0.1 \mathrm{~cm}^{-3}$. It appears that generally there is no thermalization shell around a star emitting a stellar wind.
\end{abstract}

Energiereiche Protonen des Sonnenwindes werden bei der Berührung mit kalten Wasserstoff-Atomen des interstellaren Plasmas umgeladen, d.h. neutralisiert, ohne wesentlich an kinetischer Energie einzubüßen ${ }^{1}$. Um abzuschätzen, wie lange es dauert, bis die so entstehenden schnellen Wasserstoffatome durch elastische Stöße mit dem umgebenden kalten Wasserstoffgas abgebremst sind, wurde folgende Rechnung durchgeführt.

Wir betrachten den Elementarvorgang, den Stoß zweier H-Atome und den dabei stattfindenden Energieaustausch, auf die einfachst mögliche Weise, d.h. klassisch als elastische Streuung. Für die Beschreibung von Stößen zwischen Atomen (bei bekanntem Wechselwirkungspotential) reicht das unter folgenden Bedingungen aus:

a) In einem Energiebereich der stoßenden Atome, in dem praktisch nur elastische Stöße vorkommen,

b) in einem weiteren Energiebereich, in dem Anregungs- und Ionisierungsenergien der stoßenden Atome vernachlässigbar klein gegen die gesamte übertragene Energie sind,

c) wenn typisch quantenmechanische Effekte wie Interferenzen (,,rainbow"- und ,,glory"-Streuung) oder Tunnelung vernachlässigbar sind,

d) wenn die Ungenauigkeit des Impulses $(k)$ und des Stoßparameters $(b)$ vernachlässigbar sind, d.h., wenn gilt: $(k \cdot b) \theta \gg 1$ (in atomaren Einheiten), wobei $\theta$ der Streuwinkel ist (s. Abb. 1).

Gemäß d) werden also klassische Rechnungen (auf Grund der Ungenauigkeitsrelation) bei kleinen Streuwinkeln oder fastzentralen Stößen unzureichend.

* Diese Arbeit wurde durch ein Stipendium der Deutschen Forschungsgemeinschaft ermöglicht.

1 L. Biermann u. E. Trefftz, Z. Astrophys. 49, 111 [1960].
Jedenfalls erscheint es nützlich, auch immer dann erst einmal die klassische elastische Streuung durchzurechnen, wenn man aufwendige quantenmechanische Berechnungen von Wirkungsquerschnitten auszuführen gedenkt. Das soll später noch erläutert werden.

Als Wechselwirkungspotential zwischen den beiden H-Atomen wurden die von Kolos und WolniewICZ ${ }^{2}$ berechneten $\mathrm{H}_{2}$-Potentiale für die Zustände $1 \Sigma_{\mathrm{g}}^{+}$und $3 \Sigma_{\mathrm{u}}^{+}$in der Born-Oppenheimer-Näherung benutzt.

Die Formulierung unserer Aufgabe legt es nahe, dasjenige Laborsystem auszuzeichnen, in dem ein H-Atom (das zweite) vor dem Stoß ruht.

Zunächst wollen wir im Schwerpunktsystem rechnen. Dann haben wir das dem 2-Körperproblem äquivalente Problem der Bewegung eines Teilchens (mit der reduzierten Masse $\mu$ ) in einem (zentralsymmetrischen) Potential $U$. In der Abb. 1 werden die verwendeten Größen eingeführt.

Aus der Erhaltung von Energie $E$ und Drehimpuls $M$ ergeben sich die Gleichungen

$$
\begin{aligned}
\dot{r} & =\sqrt{\frac{2}{\mu}(E-U)-\frac{M^{2}}{\mu^{2} r^{2}}}, \quad \begin{aligned}
M & =\mu r^{2} \dot{\varphi} \\
& =\mu b v_{\infty}=\text { const },
\end{aligned} \\
\mathrm{d} t & =\frac{\mathrm{d} r}{\sqrt{\frac{2}{\mu}(E-U)-\frac{M^{2}}{\mu^{2} r^{2}}}}
\end{aligned}
$$

und mit $\mathrm{d} \varphi=\left(M / \mu r^{2}\right) \mathrm{d} t$

$$
\varphi_{0}=\int_{r_{0}}^{\infty} \frac{b \frac{\mathrm{d} r}{r^{2}}}{\sqrt{1-\frac{b^{2}}{r^{2}}-\frac{U(r)}{E}}}=\int_{0}^{b / r_{0}} \frac{\mathrm{d} z}{\sqrt{1-z^{2}-\frac{U(b / z)}{E}}} .
$$

2 W. Kolos u. L. Wolniewicz, J. Chem. Phys. 43, 2429 [1965]. 


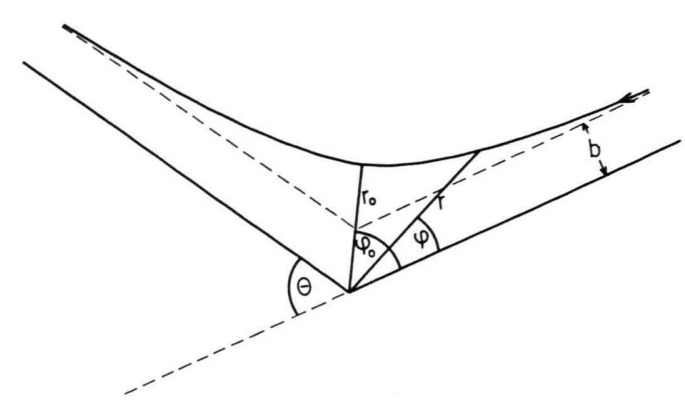

Abb. 1. Bewegung eines Teilchens in einem zentralsymmetrischen Potential.

$r_{0}$ ist die größte Nullstelle des Radikanden in (3). Aus Abb. 1 erhalten wir

$$
\theta=\left|\pi-2 \varphi_{0}\right|
$$

Bei punktweise gegebenem Potential $U(r) \operatorname{muß~(3)~}$ numerisch integriert werden. Dann ergibt sich in Abhängigkeit von der kinetischen Energie $E$ eine Funktion

$$
\theta=\theta(b, E) \quad \text { bzw. } \quad b=b(\theta, E)
$$

wenn wir nun in dem ausgezeichneten Laborsystem, in dem das zweite Teilchen vor dem Stoß ruht, anstatt des Streuwinkels die übertragene Energie einführen mit

$$
\varepsilon=\frac{\mu v^{2}}{2} \frac{4 m_{1}}{\left(m_{1}+m_{2}\right)} \sin ^{2} \frac{\theta}{2},
$$

so erhalten wir anstatt (5) eine Funktion

$$
\varepsilon=\varepsilon(b, E) \quad \text { bzw. } \quad b=b(\varepsilon, E) .
$$

Dann ist der differentielle Wirkungsquerschnitt für die Energieübertragung

$$
\mathrm{d} \sigma_{E}=2 \pi b\left|\frac{\mathrm{d} b}{\mathrm{~d} \varepsilon}\right|_{E=\text { const }} \mathrm{d} \varepsilon .
$$

Die für die Berechnung der Abbremsung signifikante „Brems"-Größe ist das Integral

$$
A=2 \pi \int \varepsilon(b, E) b \mathrm{~d} b,
$$

in der die übertragene Energie mit dem differentiellen Wirkungsquerschnitt $2 \pi b \mathrm{~d} b$ als Gewichtsfunktion multipliziert wird. In erster Näherung beschreiben wir dann die Abbremsung durch die Gleichung

$$
\frac{\mathrm{d}}{\mathrm{d} t}\left(\frac{m v^{2}}{2}\right)=-N v \cdot A,
$$

wobei $N$ die Teilchendichte des bremsenden Gases, $v$ die Geschwindigkeit der anfliegenden Atome ist.
Daraus erhalten wir die Strecke, auf der die stoßenden H-Atome im Mittel die Hälfte ihrer Energie verloren haben:

$$
\lambda=\int_{v / \sqrt{2}}^{v} \frac{m v^{\prime} \mathrm{d} v^{\prime}}{N A\left(v^{\prime}\right)} .
$$

In grober Näherung - wenn wir $A$ als konstant annehmen - ist

$$
\lambda \cong 1 / N \sigma \quad \text { mit } \quad \sigma=\left(4 A / m v^{2}\right)
$$

Hier ist $\sigma$ so definiert, daß sich für starre Kugeln der geometrische Querschnitt ergibt.

Bei der numerischen Integration von (3) wurden folgende Näherungen gemacht:

Die von Kolos und WolNIEwICZ ${ }^{2}$ berechneten Potentialkurven reichen von $r=0,4$ bis $10,0\left(1 \Sigma_{\mathrm{g}}^{+}\right)$ bzw. $r=1,0$ bis $10,0\left({ }^{3} \Sigma_{\mathrm{u}}^{+}\right)$atomare Einheiten $(r=$ Abstand der Atomkerne). Für kleinere Kernabstände und zwischen gegebenen Stützpunkten wurden weitere Stützpunkte für die elektronische Energie

$$
U_{\mathrm{e}}=U-1 / r
$$

durch Interpolation mit Polynomen 3. Grades bestimmt. Dabei wurde das erste Polynom durch einen weiteren Stützpunkt in $r=0$ mit der (experimentellen) elektronischen Energie des ,,united atom “ und dort verschwindender Steigung festgelegt. (Weiter wurde die Einschränkung gemacht, daß die Polynome 3 . Grades der ersten drei Intervalle minimale Kurvenkrümmung haben und alle Polynome in den gemeinsamen Stützpunkten bis zur 2. Ableitung übereinstimmen.) $\mathrm{Zu}$ diesem interpolierten Potential wurde das Potential der Coulombschen Kernabstoßung addiert.

Für jeden geeignet gewählten (s. u.) Stützpunkt (i) der Potentialkurve wurde aus der Gleichung $\left(1-b_{\mathrm{i}}^{2} / r_{\mathrm{i}}^{2}-U\left(r_{\mathrm{i}}\right) / E\right)=0$ ein Stoßparameter $b_{\mathrm{i}}$ bestimmt und dann von $b_{\mathrm{i}} / r_{\mathrm{i}}$ bis $b_{\mathrm{i}} / 10$ integriert. Für die stückweise analytische Integration in hinreichend kleinen Intervallen wurde der Radikand $\left(1-z^{2}-U\left(b_{\mathrm{i}} / z\right) / E\right)$ in einem sehr kleinen Intervall $\left(10^{-4}\right.$ at.E.) anschließend an die Nullstelle durch ein Polynom ersten Grades, in den folgenden Intervallen durch Polynome zweiten Grades approximiert. Weil in dem betrachteten Energiebereich Potentialwerte für $r>10$ at. E. innerhalb der Rechengenauigkeit gleich dem asymptotischen Wert gesetzt werden konnten, war es nicht nötig, die Potentialkurve für größere Abstände zu extra- 
polieren. Im Restintervall von $b_{\mathrm{i}} / r_{\max }$ bis 0 konnte der Potentialterm im Radikanden gestrichen werden.

Jede Integration von (3) ergab bei festem $b_{\mathrm{i}}$ und festem $E$ einen Punkt der Funktion $\varepsilon=\varepsilon(b, E)$. Für jedes feste $E$ mußten dann Stützpunkte $\left(b_{\mathrm{i}}, \varepsilon_{\mathrm{i}}\right)$ in geeigneter Zahl und geeigneter Plazierung bestimmt werden, um das Integral (8) mit hinreichender Genauigkeit zu erhalten.

In der Abb. 2 sind nun Funktionen $\varepsilon(b, E)$ und $b \cdot \varepsilon(b, E)$ skizzenhaft aufgetragen, wie sie typisch bei Potentialen mit einem anziehenden Bereich vorkommen. Im oberen Bild wurden noch auf der Energiekoordinate die Elektronenterme des gestoBenen Atoms skizziert. Wir sehen deutlich die Vernachlässigungen, die wir bei der klassischen elastischen Streuung machen. Im Fall I wird eine maximale Energie $E_{1}$ für $b=0$ übertragen, die kleiner als die niedrigste Anregungsenergie ist. Die elasti-

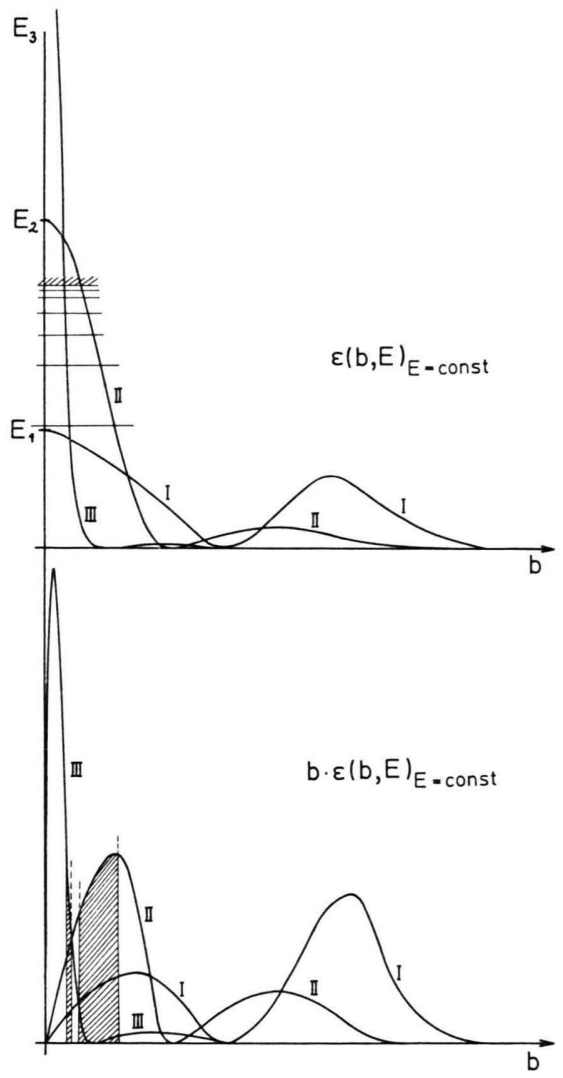

Abb. 2. Schematisierter Kurvenverlauf der übertragenen Energie $\varepsilon(b, E)$ und $b \cdot \varepsilon(b, E)$ für $E=$ const bei einem anziehenden Potential, $E_{1}<$ Anregungsenergie, $E_{3}>$ Ionisierungsenergie, $E_{2}$ dazwischen. sche Näherung scheint gerechtfertigt. Das Maximum von I im oberen Bild zeigt an, daß in diesem Bereich des Stoßparameters dem Wellencharakter der stoßenden Atome (,,rainbow"-Streuung) Rechnung getragen werden müßte.

Wenn wir in den Fällen II und III die Schnittpunkte von $\varepsilon(b)$ mit dem tiefsten Energieniveau und mit der Ionisierungsgrenze ins untere Bild projizieren, erhalten wir einen Eindruck, für welchen ungefähren Anteil von $A$ wir korrekterweise elektronische Anregungen des gestoßenen Atoms hätten berücksichtigen müssen, bzw. welcher Anteil von $A$ für eine bestimmte Energie jenseits der Ionisationsgrenze des gestoßenen Atoms liegt.

Man wird annehmen müssen, daß sich bei einer ausführlichen quantenmechanischen Rechnung die (integrale) „Bremsgröße“ $A$ ändert. Ob aber die in der klassischen elastischen Näherung berechneten $A$-Werte entweder ganz falsch oder schon ziemlich richtig sind, kann entweder durch das Experiment oder durch eben diese aufwendigen quantenmechanischen Rechnungen entschieden werden. Seit den Arbeiten von Gryzinski Ende der fünfziger Jahre ist man eher geneigt, für die klassisch gerechneten $A$-Werte eine gute Qualität selbst in dem Bereich zu erwarten, wo quantenmechanische Effekte wesentlich sind.

In der Abb. 3 (I, II, III und IV) sind einige der berechneten Funktionen $\varepsilon$ und $b \cdot \varepsilon$ für verschiedene Energien $E$ (angegeben in Geschwindigkeiten von $\mathrm{km} /$ sec des stoßenden H-Atoms) aufgezeichnet, und zwar erhalten wir im Falle des Singlett-Potentials die Funktionen in I und III, im Falle des TriplettPotentials die Funktionen in II und IV. Die Funktionen $\varepsilon$ und $b \cdot \varepsilon$ sind für die Geschwindigkeiten $40 \mathrm{~km} / \mathrm{sec}$ und $150 \mathrm{~km} / \mathrm{sec}$ vollständig, für die Geschwindigkeiten 75,100 und $200 \mathrm{~km} / \mathrm{sec}$ teilweise ausgezeichnet, um ihre Abhängigkeit von $b$ und $E$ zu zeigen. Betrachten wir zunächst die SinglettStreuungen: Um etwa $50 \mathrm{~km} / \mathrm{sec}$ und kleinere Geschwindigkeiten hat die auf das zweite H-Atom elastisch übertragene Energie ein dominierendes Maximum bei einem Stoßparameter von etwa drei atomaren Einheiten. Dieses Maximum ist bei etwa $100 \mathrm{~km} / \mathrm{sec}$ relativ zum $\varepsilon$-Wert für $b=0$ praktisch verschwunden, für $A$ ergeben sich ab $\mathbf{b}$ r noch nennenswerte Beiträge aus der Umgebung dieses Maximums bis zu etwa $150 \mathrm{~km} / \mathrm{sec}$.

Größere prozentuale Anteile von $A$, in denen die elastisch übertragene Energie zur elektronischen 


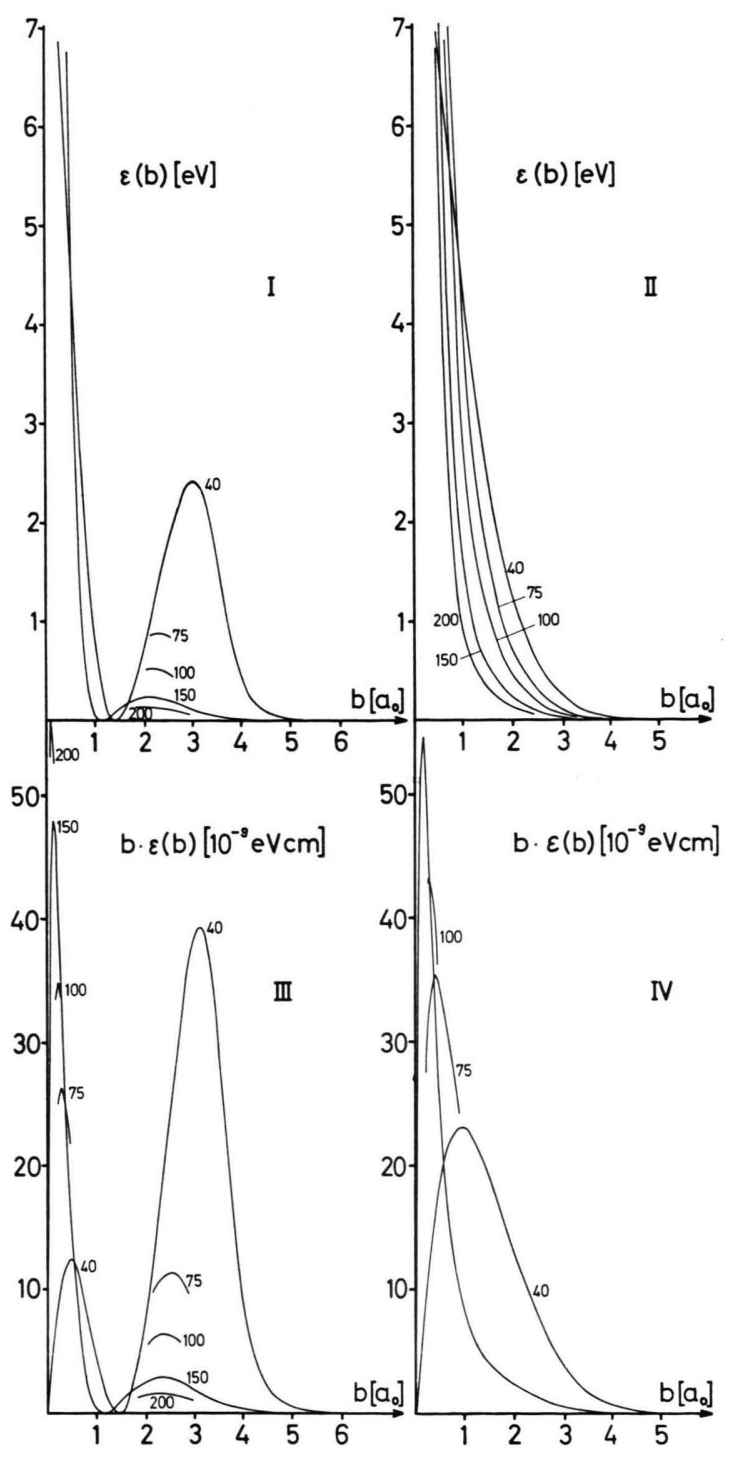

Abb. 3. Berechnete Funktion der übertragenen Energie $\varepsilon(b, E)$ und $b \cdot \varepsilon(b, E)$ für das anziehende Singlett-Potential von $\mathrm{H}_{2}$ (I bzw. III) und das abstoßende Triplett-Potential von $\mathrm{H}_{2}$ (II bzw. IV). Parameter ist die Energie, angegeben in Geschwindigkeiten $(\mathrm{km} / \mathrm{sec})$ der schnellen $\mathrm{H}$-Atome.

Anregung ausreichen würde, ergeben sich bei Geschwindigkeiten von etwa $50-120 \mathrm{~km} / \mathrm{sec}$, über $150 \mathrm{~km} / \mathrm{sec}$ kommen die wesentlichen Anteile von $A$ von Übertragungsenergien, die wesentlich über der Ionisierungsenergie des H-Atoms liegen.

Bei den Triplett-Streuungen gibt es keinen „,rainbow"-Bereich. Elektronische Anregungen scheinen relativ wichtig für $A$ bei etwa $75-180 \mathrm{~km}$ pro sec. Für größere Geschwindigkeiten werden auch wieder elastisch Energien übertragen, die wesentlich größer sind als die Ionisierungsenergie des $H$.

In der Abb. 4 sind die berechneten $A$-Werte in Abhängigkeit von der Geschwindigkeit der anfliegenden schnellen H-Atome für Singlettstreuung, Triplettstreuung, und die statistische Mischung $1: 3$ aufgetragen. Das Maximum bei $125 \mathrm{~km} / \mathrm{sec}$ sollte man nicht so ernst nehmen, weil es gerade in dem Energiebereich liegt, in dem Übertragungsenergien von der Größe der elektronischen Anregungsenergien zu $A$ den Hauptteil liefern.

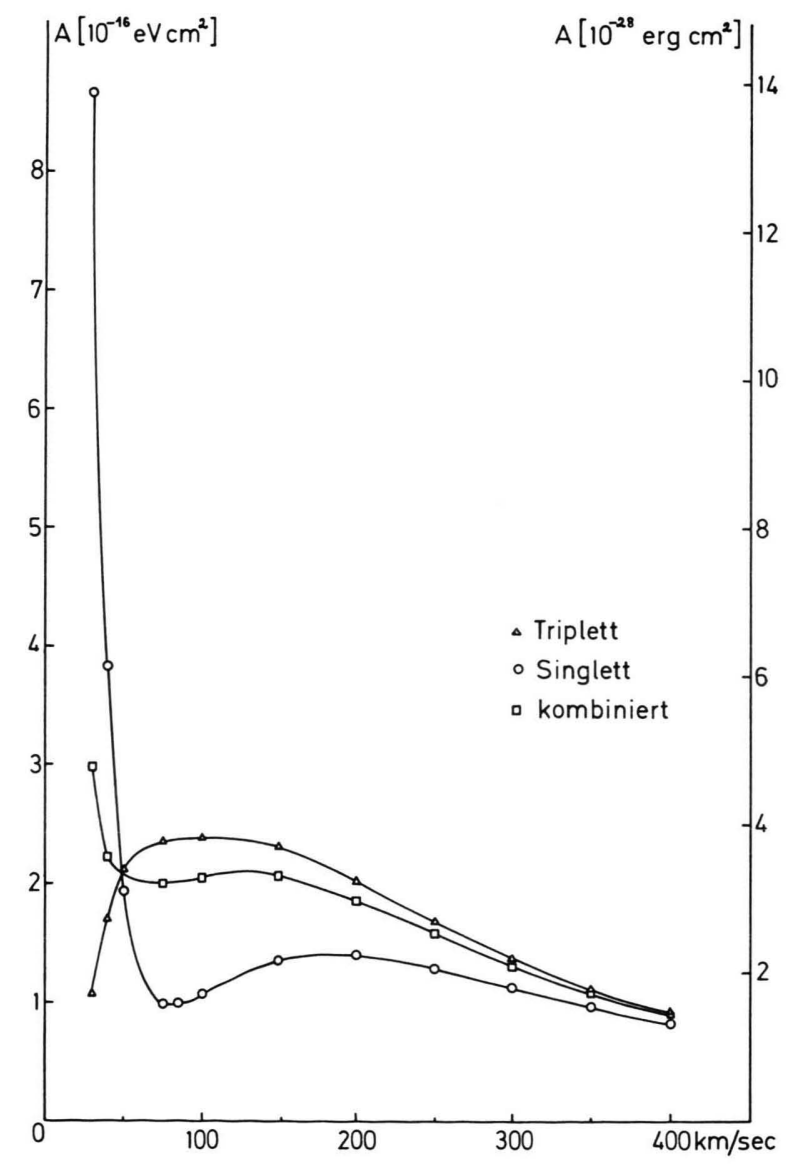

Abb. 4. Berechnete Werte der ,Bremsgröße" $A$ in Abhängigkeit von der Geschwindigkeit $(\mathrm{km} / \mathrm{sec})$ der schnellen H-Atome für Singlettstreuung, Triplettstreuung und die statistische Mischung $1: 3$.

Schließlich wurden noch nach der Formel (11) einige Abschätzungen für die Bremswege $\lambda$ berechnet. Unter der Annahme, daß die interstellare H-Dichte $\approx 0,1 \mathrm{~cm}^{-3}$ beträgt, erhalten wir die Werte der Tabelle 1. 


\begin{tabular}{rccc}
\hline $\begin{array}{c}\text { Geschw. } \\
{[\mathrm{km} / \mathrm{sec}]}\end{array}$ & $\begin{array}{c}E_{\text {kinet. }} \\
{[\mathrm{eV}]}\end{array}$ & $\begin{array}{c}\sigma \\
{\left[10^{-17} \mathrm{~cm}^{2}\right]}\end{array}$ & $\begin{array}{c}\lambda \\
{\left[10^{18} \mathrm{~cm}\right]}\end{array}$ \\
\hline 50 & 13 & 3,16 & 0,32 \\
100 & 52 & 0,79 & 1,27 \\
200 & 208 & 0,178 & 5,6 \\
300 & 470 & 0,0556 & 18,2 \\
400 & 835 & 0,0416 & 24,0 \\
\hline
\end{tabular}

Tab. 1.

Die hier ausgeführte Näherungsrechnung läßt also keinen anderen Schluß zu, als daß neutralisierte
Sonnenwindteilchen beim Übergang vom interplanetaren zum interstellaren Raum nicht wesentlich thermalisiert werden, bevor sie den nächsten Fixstern erreichen. Das stimmt überein mit der Annahme von BLUM und $\mathrm{FAHR}^{3}$, daß die elastischen $\mathrm{H}-\mathrm{H}$ Stoßquerschnitte gegen die $\mathrm{H}-\mathrm{H}^{+}$Ladungsaustauschquerschnitte in einem Modell für das Verhalten des interstellaren Wasserstoffs vernachlässigt werden können.

3 P. W. Blum u. H. J. FaHr, Astron. Astrophysics 4, 280 [1970].

\title{
Molecular Diamagnetism: Uncoupled Calculations for Two Center Systems
}

\author{
B. M. LUdwig and J. VoITLÄNDER
}

Sektion Physik und Physikalisch-Chemisches Institut der Universität München

(Z. Naturforsch. 25 a, 867-877 [1970] ; received 28 March 1970)

For $\Sigma^{1}$ molecules constructed from first row elements of the Periodic Table computations of the diamagnetic susceptibility and the nuclear magnetic shielding constants are reported. The method used is a gauge invariant approximation to the gauge dependent "uncoupled" perturbation theory in the Hartree-Fock scheme; the groundstate description considered is provided by the best limited MO functions.

Some emphysis is placed on methodological aspects and the role of $\pi$-electrons in this context; acceptable overall agreement with experiment is achieved, especially for the mean diamagnetic susceptibility. The phenomenon of antishielding occurring for fluorine and nitrogen nuclei is shown to depend critically upon the nodal structure of the state. A similar behaviour of the induced current density is not predicted by the semiclassical Thomas-Fermi theory, for which results are obtained too. This theory fails for principle reasons to explain the linear magnetic response of nonspherical systems.

\section{Introduction}

The basic quantummechanical formulation of the diamagnetic properties of molecules proceed via second order perturbation theory ${ }^{1}$. The original approaches ${ }^{2}$ use Rayleigh-Schrödinger perturbation calculus and express the second order energies $E_{2}$
a) $E_{2}^{\chi}=-\boldsymbol{B} \hat{\chi} \boldsymbol{B}$,
b) $E_{2}^{\sigma}=\boldsymbol{B} \hat{\boldsymbol{\sigma}} \mu_{N}$

$\boldsymbol{\mu}_{N}$ being the nuclear dipole, $\boldsymbol{B}$ the external field, $\hat{\boldsymbol{\chi}}$ and $\hat{\boldsymbol{\sigma}}$ the susceptibility and shielding tensors respectively, as infinite series, which appear, except for the trivial case of atoms, not to be susceptible of actual evaluation.

There have been two perturbation variation stu-

Reprint requests to: B. M. Ludwig, c/o Phys.-Chem. Institut der Universität, D-8000 München 2, Sophienstr. 11. 1 J. I. Muscher, Adv. Magn. Res. 2, 177 [1966]. - D. E. O'ReIlly, Progr. NMR Spectrosc. 2, 1 [1967].

2 J. H. van VleCk, The Theory of Electric and Magnetic dies of these quantities covering the range of molecules to be considered here, one of which - Lipscombs $^{3}$ calculations by the perturbed or coupled Hartree-Fock method - seems to be adequate, but rather involved and time consuming. A residual gauge dependence still subsists. The other ${ }^{4}$ uses different methods for the susceptibility and shielding constants, which however are manifestations of one single effect, the intramolecular Faraday effect. The work presented here may be thought of as being an extension and simplification of these procedures. It includes Thomas-Fermi molecules too and may contribute to the discussion of the role played by $\pi$-electrons.

Susceptibilities, University Press, Oxford 1932. N. F. Ramsey, Phys. Rev. 78, 699 [1950].

3 W. N. Lipscomb, Adv. Magn. Res. 2, 138 [1966].

4 H. J. Kolker and M. Karplus, J. Chem. Phys. 41, 1259 [1964]; ibid. 38, 1263 [1963], and 39, 2011 [1963]. 